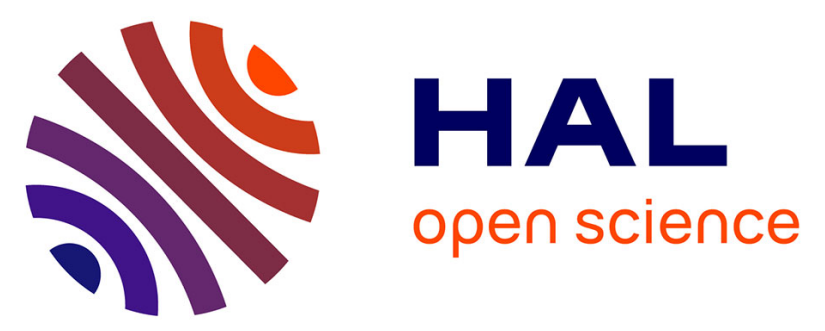

\title{
The adaptive robust lot-sizing problem with backorders under demand uncertainty
}

Paula Metzker, Simon Thevenin, Yossiri Adulyasak, Alexandre Dolgui

\section{To cite this version:}

Paula Metzker, Simon Thevenin, Yossiri Adulyasak, Alexandre Dolgui. The adaptive robust lot-sizing problem with backorders under demand uncertainty. 2021 IEEE International Conference on Automation Science and Engineering (CASE), Aug 2021, Lyon, France. 10.1109/CASE49439.2021.9551425 . hal-03375198

\section{HAL Id: hal-03375198 \\ https://hal.science/hal-03375198}

Submitted on 12 Oct 2021

HAL is a multi-disciplinary open access archive for the deposit and dissemination of scientific research documents, whether they are published or not. The documents may come from teaching and research institutions in France or abroad, or from public or private research centers.
L'archive ouverte pluridisciplinaire HAL, est destinée au dépôt et à la diffusion de documents scientifiques de niveau recherche, publiés ou non, émanant des établissements d'enseignement et de recherche français ou étrangers, des laboratoires publics ou privés. 


\title{
The adaptive robust lot-sizing problem with backorders under demand uncertainty
}

\author{
Paula Metzker ${ }^{1}$, Simon Thevenin ${ }^{1}$, Yossiri Adulyasak ${ }^{2}$ and Alexandre Doulgui ${ }^{1}$
}

\begin{abstract}
To efficiently meet demand in a production system, the lot-sizing problem determines a production plan that minimizes the overall costs, optimizes the use of the available resources, and satisfies demand requirements. Nonetheless, uncertainties in the production environment directly affect the quality and feasibility of the production plans. In fact, demand can be highly volatile and influenced by multiple factors such as age, life-cycle, economic context, reference groups, culture, festive season. To increase the robustness of the production plan to unforeseen uncertainties, one could rely on the robust optimization methodology that offers ease and flexibility to account for uncertain parameters. In the light of the robust approaches, an adaptive robust uncapacitated lot-sizing model is proposed to deal with demand uncertainty. It offers a production plan that can be updated when demand information unfolds over time. Numerical experiments demonstrate that the adaptive model can outperform the static model, while marginal additional computational effort is required to obtain a robust production plan. The results also indicate that the proposed approach is a better alternative for production planning within a system that is flexible for changes in the lot size at each period.
\end{abstract}

\section{INTRODUCTION}

The production planning activity makes the best use of resources to satisfy the production requirements over a planning horizon. It also controls the quantity and the availability of materials and components to meet a specific demand [20]. In this context, production lot-sizing decisions are crucial to balance setup and inventory costs. The classical singlelevel single-item lot-sizing problem (LSP) was introduced by Wagner and Whitin [24], and it determines the production quantities that minimize the overall costs and meets the demands. Due to its practical importance, the LSP attracted a wide range of research from the industrial engineering and operations research communities [2], [3], [17], [22]. While most studies consider the deterministic LSPs, the demand is not known with certainty when the production is planned. Even though a significant number of studies have considered demand uncertainty in lot-sizing [16], there are still important gaps in the literature. In particular, few works address the adaptive framework, where unknown parameters unfold at each period, and the production quantity is updated to react to this information. Recent adaptive robust optimization approaches, which rely on limited information, have proven to be effective in several problems [19], [10], [9]. Our study aims to explore the efficiency and effectiveness of the adaptive robust optimization techniques for the LSP under demand uncertainty.

\footnotetext{
${ }^{1}$ IMT Atlantique, LS2N - UMR CNRS 6004, F-44307 Nantes, France

${ }^{2}$ HEC Montreal, Montreal, Canada
}

Robust optimization [7] considers the representation of uncertainty in the form of an uncertainty set, and its main objective is to minimize the total cost under the worst-case realization. A simple uncertainty set, called box uncertainty set, was introduced by Soyster et al. [23], and it limits the uncertain parameter to an interval of possible values bounded by the minimum and the maximum values that the uncertainty can achieve. However, the box uncertainty set tends to provide an overly conservative solution. Therefore, Bertsimas and Sim [11], [12] propose the box polyhedral uncertainty set, where the uncertain parameter is bounded within a range of values whose size is controlled by the decision-maker through a risk aversion index called budget $\Gamma$. The budget limits the maximum deviation of uncertain parameters from their nominal values. Therefore, a large budget considers large deviations from the average, which leads to more conservative decisions. Hence, the choice of the uncertainty set is crucial to improve the performance and mitigate the uncertainties.

Bertsimas and Thiele [13], [14] propose a static robust formulation of the lot-sizing problem with backorders based on the budgeted-uncertainty set proposed by Bertsimas and Sim [11], [12]. For the adaptive framework, Melamed et al. [18] propose an adaptive robust LSP under demand uncertainty based on the affinely adjustable robust counterpart. They describe the adaptive variables by linear decision rules, and solve the adaptive robust counterpart reformulation via reformulation per constraint and dualization approach. However, their model considers the box uncertainty set, that can lead to conservative solutions. In this paper, we propose an adaptive robust LSP model based on the budgeted uncertainty set to improve the quality of the production plan. The resulting method maintains robust, and it has its conservatism controlled with regard to an fixed risk acceptance.

The remainder of this paper is organized as follows: Section II introduces the lot-sizing problem. Section III presents an overview of the robust optimization methodology, the static lot-sizing model based on the budgeted uncertainty set proposed by Bertsimas and Thiele [13], and the adaptive robust lot-sizing model with backorders suggested in this paper. Section IV provides the numerical experiments and a comparison of the static and adaptive robust plans. Finally, Section V summarizes the main findings of this work.

\section{PROBLEM STATEMENT}

The single-level single-item LSP with backordering determines the production setup and quantity in a finite horizon $T=\{1, \ldots,|T|\}$, to minimize the overall costs, and to meet 
the demand. For each period $t$, the following parameters are given: $s_{t}$ the setup cost, $v_{t}$ the unit production cost, $h_{t}$ the unit inventory cost, $b_{t}$ the unit backordering cost, and finally $d_{t}$ the demand in period $t$. For each period $t$, the following decision variables are used: $X_{t}$, the quantity to produce, $I_{t}$, the inventory level, $B_{t}$, the backordering level at the end of the period, and $Y_{t}$ the setup decision.

The deterministic formulation of the LSP is as follows:

$$
\begin{array}{ll}
\min \sum_{t \in T}\left(s_{t} Y_{t}+v_{t} X_{t}+h_{t} I_{t}+b_{t} B_{t}\right) & \\
\text { s.t. : } & \\
I_{t}-B_{t}=I_{t-1}-B_{t-1}+X_{t}-d_{t} & \forall t \in T \\
X_{t} \leq M_{t} \cdot Y_{t} & \forall t \in T \\
X_{t}, I_{t}, B_{t} \geq 0 & \forall t \in T \\
Y_{t} \in\{0,1\} & \forall t \in T
\end{array}
$$

Without loss of generality, we assume that the inventory and the backordering levels at the beginning of the production planning are zero. The objective function (1) minimises the overall costs with regard to the setup, unit production, inventory and backordering costs. Constraints (2) are the inventory balance equations. Here, the production in the current period $t$ and the inventory from the previous period $t-1$ are used to meet the demand $d_{t}$ and the backordered quantity from period $t-1$. If the amount of goods available is not sufficient to meet the demand, then the amount of missing goods is captured by the backorder variable $\left(B_{t}\right)$. Any remaining amount of goods after satisfying both the demand and the items backordered before, if any, is kept in stock $\left(I_{t}\right)$. Constraints (3) are setup-forcing constraints that relate the production quantities $\left(X_{t}\right)$ to the setup decisions $\left(Y_{t}\right)$. These constraints set the setup variable to $1\left(Y_{t}=1\right)$ if any production is incurred in period $t$ and the setup remains inactive otherwise $\left(Y_{t}=0\right)$. Although this paper considers the uncapacitated problem, for which we should set $M_{t}$ as a big number, we can define a natural bound for the lot size in period $t$ given by $M_{t}=\sum_{t \in T} d_{t}$.

The deterministic problem does not take into account uncertainties. Thus, this model leads to a suboptimal or even an unrealistic plan in an uncertain context. Although classical techniques such as Rolling Horizon and Model Predictive Control can be applied within an adaptive framework, updating decisions according to information revealed over time, these approaches do not immunize the system against the occurrence of unexpected scenarios, such as as when uncertainty assumes the worst possible value. Thus, decision makers would like to have a method that efficiently meets the demands and mitigates these uncertainties in a robust and reliable way. Robust adaptive optimization emerges as a promising methodology that takes demand uncertainties into account, proposing a production plan that can be updated over time, while still providing some insight into the optimal production plan over the production horizon.

\section{ADJUSTABLE ROBUST OPTIMIZATION METHODOLOGY}

The robust optimization protects the decision system from uncertainties through an optimal decision that remains viable for the worst case realization of the uncertain parameter. The static robust optimization (RO) [4] determines the decisions in the presence of uncertainty, and no recourse action is allowed after the realization of the uncertain parameters. Based on the worst case perspective of the uncertain parameters, the approach is highly conservative to ensure the feasibility of the production plan to any realization of the uncertainty. The RO method reformulates the non-deterministic problem as a robust counterpart, where the uncertainty is described with an uncertainty set. Ben-Tal et al. [4] indicates that there are three approaches to solve the $\mathrm{RC}$, that are: reformulation per constraint, dualization and adversarial approach.

The static RO does not account for the dynamic of the decision process. The Adjustable Robust Optimization (ARO) [5] is an extension of the RO that considers uncertainties revealed over time during the execution, and allows the decision maker to adjust some of the decisions according to the current knowledge of the revealed data. Therefore, the recourse decisions are commonly described as a function of the (upcoming) realizations of the unknown parameters. Since such reformations are usually intractable, a common approach is to adopt the affine decisions rule (or affine function) [1]. Under these affine polices, the adjustable decisions are linearly dependent on the realization of the uncertain parameter [21]. As a result, an optimal affine policy can be efficiently computed by solving a convex optimization problems as linear, quadratic, conic or semidefinite optimization problem [8].

Under the affinely adjustable formulation, we can rely on the common solution approaches for the robust optimization to solve the problem. This work relies on the reformulation per constraint and dualization approach [6], [7], [4] to solve the adaptive robust problem. This approach follows three steps. First, we reformulate each constraint subject to the uncertainty as a worst case problem. Then, we dualize the reformulated problem. Finally, we replace the reformulated problems by their duals. Thus, we obtain a convex robust counterpart problem that can be handled by common commercial solvers. In the subsequent section, the static robust LSP based on the budgeted uncertainty, which was first introduced by Bertsimas and Thiele [13], followed by the adaptive robust model proposed in this paper are presented.

\section{A. Static robust optimization LSP formulation}

Bertsimas and Thiele [13], [14] propose a static robust formulation of the LSP with setup and backordering based on the budgeted-uncertainty set proposed by Bertsimas and Sim [12]. Before applying the robust methodology to the problem, the authors reformulate the inventory balance constraints (2) as a pair of inequalities based on the piecewise linearity and convexity of the inventory and backordering costs. Then, they represent the uncertain demand $\widetilde{d}$ with the budgeted uncertainty set $\mathcal{U}$, such that the conservatism of the robust 
solution can be controlled by the risk aversion represented by the budget $\Gamma$. The budgeted uncertainty set representing the uncertain demand is given by $\mathcal{U}=\left\{\widetilde{\Gamma} \in \mathbb{R}^{\sqcup}: \widetilde{\Gamma}=\right.$ $\bar{\Gamma} \sqcup+\ddagger \sqcup \hat{\Gamma} \sqcup \sqcup \in \mathcal{T} ;-\infty \leq \ddagger \sqcup \leq \infty \sqcup \in \mathcal{T} ; \sum_{\tau=\infty}^{\sqcup}|\ddagger \tau| \leq$ $-\sqcup\}$, where $\bar{d}$ is the average demand, and $\hat{d}$ its standard deviation. Finally, they express the robust model in terms of the uncertain parameter as a robust counterpart model. Here, we can represent a natural limit for the lot size based on the highest possible value of the demand for each period $t$, that is, $\max d_{t}=\bar{d}_{t}+\hat{d}_{t}$. Thus, for the LSP under demand uncertainty, $\mathbf{M}$ is set to $M=\sum_{t \in T}\left(\bar{d}_{t}+\hat{d}_{t}\right)$.

Using a reformulation per constraint approach to handle the robust counterpart problem, they reformulate the static robust lot-sizing problem which yields the following mixedinteger model:

$$
\begin{array}{lr}
\min \sum_{t \in T}\left(s_{t} Y_{t}+v_{t} X_{t}+H_{t}\right) & \\
\text { s.t. : } & \\
H_{t} \geq h_{t}\left(\Gamma_{t} \lambda_{t}+\sum_{\tau=1}^{t}\left(X_{j}-\bar{d}_{j}+\mu_{t}^{\tau}\right)\right) & \forall t \in T \\
H_{t} \geq-b_{t}\left(-\Gamma_{t} \lambda_{t}+\sum_{\tau=1}^{t}\left(X_{j}-\bar{d}_{j}-\mu_{t}^{\tau}\right)\right) & \forall t \in T \\
X_{t} \leq M \cdot Y_{t} & \forall t \in T \\
\lambda_{t}+\mu_{t}^{\tau} \geq \hat{d}_{t} & \forall t \in T ; \tau \leq t \\
X_{t}, I_{t}, B_{t}, \lambda_{t} \geq 0 & \forall t \in T \\
\mu_{t}^{\tau} \geq 0 & \forall t \in T ; \tau \leq t \\
Y_{t} \in 0,1 & \forall t \in T
\end{array}
$$

\section{B. Adaptive robust optimization LSP formulation}

This section introduces the proposed ARO formulation for the LSP within the adaptive decision strategy. In this framework, the setups are fixed at the beginning of the planning horizon, but the lot sizes and the costs incurred from the inventory management are updated as the actual demand are known over time. Similar to the static framework, it is possible to consider the inventory and the backorder quantities indirectly by their costs, represented by $H_{t}$. Here, as both production quantities and inventory costs depend on the uncertain demand, they become adjustable variables.

To ensure the constraint-wise formulation of the robust optimization methodology, the robust objective function is now placed as a constraint which determines the worst-case cost. In addition, the affine decision rules (ADR) are used to represent the dependence of the adjustable variables up to the period $t$ on the revealed demand (from $d_{1}$ to $d_{t-1}$ ), such that the variables are parametrized as follows:

$$
X_{t}(\widetilde{d})=X_{t}^{0}+\sum_{\tau=1}^{t-1} X_{t}^{\tau} d_{\tau} \quad H_{t}(\widetilde{d})=H_{t}^{0}+\sum_{\tau=1}^{t-1} H_{t}^{\tau} d_{\tau}
$$

The following mixed-integer affinely linear adaptive robust counterpart model is obtained: $\min \mathbf{F}$

$$
\begin{aligned}
& \text { s.t. : } \\
& F \geq \sum_{t \in T}\left[s_{t} Y_{t}+v_{t} X_{t}^{0}+H_{t}^{0}\right. \\
& \left.+\max _{Z \in \mathcal{U}} \sum_{\tau=1}^{t-1}\left[\left(\bar{d}_{\tau}+\hat{d}_{\tau} Z_{\tau}\right)\left(v_{t} X_{t}^{\tau}+H_{t}^{\tau}\right)\right]\right] \\
& H_{t}^{0} \geq \max _{Z \in \mathcal{U}}\left\{-\sum_{\tau=1}^{t-1}\left(\bar{d}_{\tau}+\hat{d}_{\tau} Z_{\tau}\right) H_{t}^{\tau}+\right. \\
& \left.\sum_{\tau=1}^{t} h_{t}\left[X_{\tau}^{0}+\sum_{r=1}^{\tau-1}\left(\bar{d}_{r}+\hat{d}_{r} Z_{r}\right) X_{\tau}^{r}-\left(\bar{d}_{\tau}+\hat{d}_{\tau} Z_{\tau}\right)\right]\right\} \quad t \in T \\
& H_{t}^{0} \geq \max _{Z \in \mathcal{U}}\left\{-\sum_{\tau=1}^{t-1}\left(\bar{d}_{\tau}+\hat{d}_{\tau} Z_{\tau}\right) H_{t}^{\tau}-\right. \\
& \left.\sum_{\tau=1}^{t} b_{t}\left[X_{\tau}^{0}+\sum_{r=1}^{\tau-1}\left(\bar{d}_{r}+\hat{d}_{r} Z_{r}\right) X_{\tau}^{r}-\left(\bar{d}_{\tau}+\hat{d}_{\tau} Z_{\tau}\right)\right]\right\} \quad t \in T \\
& X_{t}^{0}+\max _{Z \in \mathcal{U}}\left[\sum_{\tau=1}^{t-1}\left(\bar{d}_{\tau} X_{t}^{\tau}+\hat{d}_{\tau} X_{t}^{\tau} Z_{\tau}\right)\right] \leq M Y_{t} \quad t \in T \\
& X_{t}^{0}+\min _{Z \in \mathcal{U}}\left[\sum_{\tau=1}^{t-1}\left(\bar{d}_{\tau} X_{t}^{\tau}+\hat{d}_{\tau} X_{t}^{\tau} Z_{\tau}\right)\right] \geq 0 \quad \forall t \in T \\
& H_{t}^{0}+\min _{Z \in \mathcal{U}}\left[\sum_{\tau=1}^{t-1}\left(\bar{d}_{\tau} H_{t}^{\tau}+\hat{d}_{\tau} H_{t}^{\tau} Z_{\tau}\right)\right] \geq 0 \quad t \in T \\
& X_{t}^{0}, H_{t}^{0} \geq 0 \\
& t \in T \\
& X_{t}^{\tau}, H_{t}^{\tau} \in \mathbb{R} \\
& t \in T ; \tau \leq t-1 \\
& Y_{t} \in\{0,1\} \\
& t \in T
\end{aligned}
$$

Applying the reformulation per constraint and dualization approach to each set of constraints associated with the uncertain demand parameters, we obtain the following mixedinteger linear adaptive robust counterpart problem:

\section{$\min \mathbf{F}$}

$$
\begin{aligned}
& \text { s.t. : } \\
& F \geq \sum_{t \in T}\left[s_{t} Y_{t}+v_{t} X_{t}^{0}+H_{t}^{0}\right. \\
& \left.+\Gamma_{t} \gamma_{t}+\sum_{\tau=1}^{t-1}\left(\bar{d}_{\tau}\left(v_{t} X_{t}^{\tau}+H_{t}^{\tau}\right)+\alpha_{t}^{\tau}+\delta_{t}^{\tau}\right)\right] \\
& H_{t}^{0} \geq \Gamma_{t} \lambda_{t}-\sum_{\tau=1}^{t-1} \bar{d}_{\tau} H_{t}^{\tau} \\
& +\sum_{\tau=1}^{t}\left[h_{t}\left(X_{\tau}^{0}-\bar{d}_{\tau}+\sum_{r=1}^{\tau-1} \bar{d}_{r} X_{\tau}^{r}\right)+\mu_{t}^{\tau}+\xi_{t}^{\tau}\right] \quad \forall t \in T \\
& H_{t}^{0} \geq \Gamma_{t} \psi_{t}-\sum_{\tau=1}^{t-1} \bar{d}_{\tau} H_{t}^{\tau} \\
& -\sum_{\tau=1}^{t}\left[b_{t}\left(X_{\tau}^{0}-\bar{d}_{\tau}+\sum_{r=1}^{\tau-1} \bar{d}_{r} X_{\tau}^{r}\right)+\varpi_{t}^{\tau}+\varepsilon_{t}^{\tau}\right] \quad \forall t \in T \\
& X_{t}^{0}+\eta_{t} \Gamma_{t}+\sum_{\tau=1}^{t-1}\left(\bar{d}_{\tau} X_{t}^{\tau}+\beta_{t}^{\tau}+\theta_{t}^{\tau}\right) \leq M Y_{t} \quad \forall t \in T \\
& X_{t}^{0}-\phi_{t} \Gamma_{t}+\sum_{\tau=1}^{t-1}\left(\bar{d}_{\tau} X_{t}^{\tau}-\pi_{t}^{\tau}+\chi_{t}^{\tau}\right) \geq 0 \quad \forall t \in T
\end{aligned}
$$




$$
\begin{aligned}
& H_{t}^{0}-\epsilon_{t} \Gamma_{t}+\sum_{\tau=1}^{t-1}\left(\bar{d}_{\tau} H_{t}^{\tau}-\sigma_{t}^{\tau}+\kappa_{t}^{\tau}\right) \geq 0 \\
& \mu_{t}^{t}-\varepsilon_{t}^{t}+\mu_{t}^{\prime t}-\varepsilon_{t}^{t}=-h_{t} \hat{d}_{t} \\
& \lambda_{t}-\mu_{t}^{t^{\prime}}-\varepsilon_{t}^{t^{\prime}} \geq 0 \\
& \varpi_{t}^{t}-\xi_{t}^{t}+\varpi_{t}^{\prime}-\xi_{t}^{\prime}=b_{t} \hat{d}_{t} \\
& \psi_{t}-\varpi_{t}^{\prime t}-\xi_{t}^{\prime} \geq 0 \\
& \alpha_{t}^{\tau}-\delta_{t}^{\tau}+\alpha_{t}^{\prime} \tau-\delta_{t}^{\prime} \tau=\hat{d}_{\tau}\left(v_{t} X_{t}^{\tau}+H_{t}^{\tau}\right) \\
& \gamma_{t}-\alpha_{t}^{\prime}{ }^{\tau}-\delta_{t}^{\prime} \tau=\hat{d}_{\tau}\left(v_{t} X_{t}^{\tau}+H_{t}^{\tau}\right) \\
& \beta_{t}^{\tau}-\theta_{t}^{\tau}+\beta_{t}^{\prime \tau}-\theta_{t}^{\prime} \tau=\hat{d}_{\tau} X_{t}^{\tau} \\
& \eta_{t}-\beta_{t}^{\prime} \tau-\theta_{t}^{\prime} \tau \geq 0 \\
& -\pi_{t}^{\tau}+\chi_{t}^{\tau}-\pi_{t}^{\prime} \tau+\chi_{t}^{\prime} \tau=\hat{d}_{\tau} X_{t}^{\tau} \\
& \phi_{t}-\pi_{t}^{\prime} \tau-\chi_{t}^{\prime} \tau \geq 0 \\
& -\sigma_{t}^{\tau}+\kappa_{t}^{\tau}-\sigma_{t}^{\prime}{ }^{\prime}+\kappa_{t}^{\prime}{ }^{\tau}=\hat{d}_{\tau} H_{t}^{\tau} \\
& \epsilon_{t}-\sigma_{t}^{\prime} \tau-\kappa_{t}^{\prime} \tau \geq 0 \\
& \mu_{t}^{\tau}-\varepsilon_{t}^{\tau}+\mu_{t}^{\prime \tau}-\varepsilon_{t}^{\prime \tau}= \\
& -\hat{d}_{\tau}\left(H_{t}^{\tau}+h_{t}-h_{t} X_{t}^{\tau}-\sum_{r=\tau+1}^{t-1} \hat{d}_{\tau} X_{r}^{\tau}\right) \\
& \lambda_{t}-\mu_{t}^{\prime} \tau-\varepsilon_{t}^{\prime} \tau \geq 0 \\
& \varpi_{t}^{\tau}-\xi_{t}^{\tau}+\varpi_{t}^{\prime} \tau-\xi_{t}^{\prime \tau}= \\
& -\hat{d}_{\tau}\left(H_{t}^{\tau}-b_{t}+b_{t} X_{t}^{\tau}+\sum_{r=\tau+1}^{t-1} \hat{d}_{\tau} X_{r}^{\tau}\right) \\
& \psi_{t}-\varpi_{t}^{\prime} \tau-\xi_{t}^{\prime} \tau=0 \\
& \alpha_{t}^{\tau}, \alpha_{t}^{\prime}{ }^{\tau}, \beta_{t}^{\tau}, \beta_{t}^{\prime \tau}, \delta_{t}^{\tau}, \delta_{t}^{\prime}{ }^{\tau}, \kappa_{t}^{\tau}, \kappa_{t}^{\prime}{ }^{\tau} \geq 0 \\
& \kappa_{t}^{\tau}, \kappa_{t}^{\prime} \tau, \mu_{t}^{\tau}, \mu_{t}^{\prime}{ }^{\tau}, \chi_{t}^{\tau}, \chi_{t}^{\prime}{ }^{\tau}, \xi_{t}^{\tau}, \xi_{t}^{\prime} \tau \geq 0 \\
& \theta_{t}^{\tau}, \theta_{t}^{\prime} \tau, \varepsilon_{t}^{\tau}, \varepsilon_{t}^{\prime} \tau, \varpi_{t}^{\tau}, \varpi_{t}^{\prime \tau}, \sigma_{t}^{\tau}, \sigma_{t}^{\prime} \tau \geq 0 \\
& \gamma_{t}, \lambda_{t}, \psi_{t}, \eta_{t}, \epsilon_{t}, \phi_{t} \geq 0 \\
& X_{t}^{0}, H_{t}^{0} \geq 0 \\
& X_{t}^{\tau}, H_{t}^{\tau} \in \mathbb{R} \\
& Y_{t} \in\{0,1\}
\end{aligned}
$$

$\forall t \in T$

$\forall t \in T$

$\forall t \in T$

$\forall t \in T$

$\forall t \in T$

$\forall t \in T ; \tau \leq t-1$

$\forall t \in T ; \tau \leq t-1$

$\forall t \in T ; \tau \leq t-1$

$\forall t \in T ; \tau \leq t-1$

$\forall t \in T ; \tau \leq t-1$

$\forall t \in T ; \tau \leq t-1$

$\forall t \in T ; \tau \leq t-1$

$\forall t \in T ; \tau \leq t-1$

$\forall t \in T ; \tau \leq t-1$

$\forall t \in T ; \tau \leq t-1$

$\forall t \in T ; \tau \leq t-1$

$\forall t \in T ; \tau \leq t-1$

$\forall t \in T ; \tau \leq t-1$

$\forall t \in T ; \tau \leq t-1$

$\forall t \in T ; \tau \leq t-1$

$\forall t \in T$

$\forall t \in T$

$\forall t \in T ; \tau \leq t-1$

$\forall t \in T$

\section{NUMERICAL EXPERIMENTS}

The experiments are performed with instances generated following the standard approach in the literature for LSP problems, as presented by Brandimarte [15]. Thus, the production cost, the holding cost, the average demand, and the standard deviation of the demand were randomly drawn from an uniform distribution within the following intervals $v_{t} \in U(20,50), h_{t} \in U(1,10), \bar{d}_{t} \in U(140,480)$, and $\hat{d}_{t} \in U(30,50)$, respectively. The setup costs are computed with the time between order formula: $s_{t}=\frac{\bar{D}_{t} \cdot T B O^{2} \cdot h_{t}}{2}$, where $\bar{D}_{t}$ represents the average demand up to period t. Instances with 10,30 , and 50 periods, a time between orders of one period, and a backorder cost equals to 2,5 , or 10 times the holding cost for each period $t$ are considered. It leads to 9 different instances. To investigate the impact of robust optimization for lot-sizing, we evaluate the performance of the methods through a simulation. For each instance, 5000 scenarios based on the Monte Carlo sampling are simulated, where the demand is drawn from a uniform distribution with support between $\bar{d}_{t}-\hat{d}_{t}$ and $\bar{d}_{t}+\hat{d}_{t}$. The problems were implemented in Python programming language, and the models solved with CPLEX 12.9.

Table I shows the expected value of perfect information (EVPI), which gives the average performance for the deterministic solution in case of perfect information of the occurrence of the uncertain demand, the nominal problem (DET), the static robust model $\left(R O_{\Gamma}\right)$ and the proposed adaptive robust model $\left(A R O_{\Gamma}\right)$. The robust model with $\Gamma$ equals 0 corresponds to the nominal problem. On the contrary, when $\Gamma$ equals $T$, this is the conservative case which is equivalent to the box uncertainty set. Note that $P C^{*}$ is an abbreviation for percentile. Table I summarises the average of the expect cost, the $95^{\text {th }}$ percentile cost, the $99^{\text {th }}$ percentile cost, and the worst case cost computed in the simulation. In addition, Table I presents the average of the objective value obtained via the respective optimization approaches for each model, and the average computational time in seconds. The results are analysed following two axes: a comparison of the nominal and the static robust LSP under demand uncertainty, then an analysis of the quality of the robust solution within the static and adaptive frameworks.

TABLE I

AVERAGE AND WORST CASE ANALYSIS OF THE LSP MODELS

\begin{tabular}{ccrrrrc}
\hline Model & Exp. Cost & $95^{\text {th }} P C^{*}$ & $99^{t h} P C^{*}$ & Worst Cost & Obj. Cost & Time(s) \\
\hline EVPI & 324,830 & 330,894 & 333,386 & 336,292 & & \\
DET & 341,056 & 372,727 & 389,093 & 412,495 & 325,006 & 1.26 \\
$R O_{\Gamma=0}$ & 341,056 & 372,727 & 389,093 & 412,495 & 325,006 & 0.02 \\
$R O_{\Gamma=1}$ & 338,604 & 363,309 & 378,598 & 401,141 & 334,346 & 0.02 \\
$R O_{\Gamma=0.2 T}$ & 340,890 & 352,178 & 360,318 & 373,033 & 365,855 & 0.02 \\
$R O_{\Gamma=0.3 T}$ & 349,853 & 362,194 & 368,971 & 379,720 & 398,046 & 0.02 \\
$R O_{\Gamma=T}$ & 392,804 & 407,655 & 415,044 & 425,638 & 504,559 & 0.03 \\
$A R O_{\Gamma=0}$ & 341,056 & 372,727 & 389,093 & 412,495 & 325,006 & 67.31 \\
$A R O_{\Gamma=1}$ & 337,478 & 356,480 & 367,547 & 381,438 & 343,217 & 163.64 \\
$A R O_{\Gamma=0.2 T}$ & 338,383 & 342,760 & 345,962 & 350,228 & 362,604 & $1,213.87$ \\
$A R O_{\Gamma=0.3 T}$ & 339,225 & 342,570 & 344,118 & 346,285 & 369,798 & $1,216.51$ \\
$A R O_{\Gamma=T}$ & 339,039 & 342,945 & 344,518 & 346,261 & 373,589 & 64.58 \\
\hline Average & 343,690 & 359,930 & 368,812 & 381,460 & 366,094 & 247.93 \\
\hline
\end{tabular}

From a robust perspective, a production plan is said to be immunized against uncertainty if its objective cost covers any realization of the overall costs incurred in the production system once the uncertainties are revealed. The results in Table I shows that the nominal model fails to mitigate the uncertainties as its objective value does not give any information neither on the average worst case cost nor on the average expected cost. Within the static framework, the robust model fails to protect against the demand uncertainty for $\Gamma$ lower than $0.2 T$. For $\Gamma$ lower than 1 , the static robust plan fails to react to the realized demands, as its optimal objective value is lower than its simulated expected cost. However, the static worst case cost for $\Gamma$ equals 1 is lower than the worst case cost incurred in the nominal model, indicating that even if the model fails to immunize the system from uncertainties, the robust model does take into account the uncertainties, and it propose better plans than a deterministic model that neglects the unexpected events. For $\Gamma$ equals $0.2 \mathrm{~T}$, the objective value covers the $95_{t h}$ percentile realization of the uncertain parameter. For this budget, the worst case scenarios are still not covered, and the robust model fails to efficiently hedge against uncertainties. However, for $\Gamma$ 
greater or equals to $0.3 T$, the static production plan remains robust, and it fully achieves its role of protecting the system from the uncertain demand.

It is also interesting to investigate the benefit of the adaptive strategy. From Table I, we can also compare the static $R O_{\Gamma}$ and the adaptive $A R O_{\Gamma}$ robust model for the same budget values $\Gamma$. Even though the adaptive model requires much more time to solve the problem than the nominal and the static robust model, its computational time remains acceptable, since the CPU time is approximately 1015 minutes. On the one hand, the case of perfect information EVPI provides a lower bound of the costs of the optimal production plan. On the other hand, the static robust models yield an upper bound on these costs. For any $\Gamma$, the ARO model outperforms the RO model, since the average adaptive expected costs, worst case cost and objective values are 4.2 $\%, 13 \%$ and $4.5 \%$ lower than the respective static averages. It indicates that the adaptive model provides a better solution than the static model within an adaptive strategy. Even if the computing time for the adaptive model is generally longer than that of the static model, the cost savings justify the longer time to obtain a plan. Furthermore, as the adaptive model determines the adaptive decisions according to the affine functions, it provides some insight into the predicted lot size quantity $\left(X_{t}^{0}\right)$ and predicted inventory management costs $\left(H_{t}^{0}\right)$ for each period $t$ along the production horizon. Consequently, the adaptive robust plans provide more indicators to deepen the understanding of the decision system. Thus, the decision makers would dispose more significant information available to support their analysis in the medium term (e.g.: over all the production horizon), even if the production plans must be updated at each production period.

\section{CONCLUSIONS}

This paper proposes an adaptive robust single-item multiperiod uncapacitated lot-sizing problem with backordering and uncertain demand. Compared to the existing literature on this topic, the performance of the robust methodology was improved by addressing the problem under the budgeted uncertainty set. A comparison between the quality of the production plan within the static and adaptive framework was also presented.

The computational experiments indicates that the adaptive robust model for the LSP under demand uncertainty could determine good solutions for an adaptive decision strategy, and offers a production plan that is both robust and adjustable to the decision context, while the production system immune to any realizations of demand uncertainty. It was showed that the adaptive model generally outperforms the static model even within the static framework, when some risk are accepted to obtain a robust production plan. In addition, an in-depth comparison between the box and the budgeted uncertainty sets in the robust static and adaptive LSP models could give some insights for decision makers to adopt a solution that is robust and closer to real-world applications. Further studies are needed to extend the adaptive model to the capacitated and multi-item version of the problem.

\section{ACKNOWLEDGMENT}

The authors wish to thank the Region Pays de la Loire and the Canada Research Chain in Supply Chain Analytics for financial support of this research.

\section{REFERENCES}

[1] B.-T. Aharon, G. Boaz, and S. Shimrit, "Robust multi-echelon multiperiod inventory control," European Journal of Operational Research, vol. 199, no. 3, pp. 922-935, 2009.

[2] I. Barany, T. Van Roy, and L. A. Wolsey, "Uncapacitated lot-sizing: The convex hull of solutions," in Mathematical Programming at Oberwolfach II. Springer, 1984, pp. 32-43.

[3] G. Belvaux and L. A. Wolsey, "A specialized branch-and-cut system for lot-sizing problems," Management Science, vol. 46, no. 5, pp. 724738, 2000.

[4] A. Ben-Tal, L. El Ghaoui, and A. Nemirovski, Robust optimization. Princeton University Press, 2009, vol. 28.

[5] A. Ben-Tal, A. Goryashko, E. Guslitzer, and A. Nemirovski, "Adjustable robust solutions of uncertain linear programs," Mathematical Programming, vol. 99, no. 2, pp. 351-376, 2004.

[6] A. Ben-Tal and A. Nemirovski, "Robust solutions of uncertain linear programs," Operations research letters, vol. 25, no. 1, pp. 1-13, 1999.

[7] $\_$, "Robust solutions of linear programming problems contaminated with uncertain data," Mathematical Programming, vol. 88, no. 3, pp. 411-424, 2000.

[8] D. Bertsimas and H. Bidkhori, "On the performance of affine policies for two-stage adaptive optimization: a geometric perspective," Mathematical Programming, vol. 153, no. 2, pp. 577-594, 2015.

[9] D. Bertsimas and F. J. de Ruiter, "Duality in two-stage adaptive linear optimization: Faster computation and stronger bounds," INFORMS Journal on Computing, vol. 28, no. 3, pp. 500-511, 2016.

[10] D. Bertsimas, E. Litvinov, X. A. Sun, J. Zhao, and T. Zheng, "Adaptive robust optimization for the security constrained unit commitment problem," IEEE transactions on power systems, vol. 28, no. 1, pp. 52-63, 2012.

[11] D. Bertsimas and M. Sim, "Robust discrete optimization and network flows," Mathematical programming, vol. 98, no. 1-3, pp. 49-71, 2003.

[12] - "The price of robustness," Operations Research, vol. 52, no. 1, pp. 35-53, 2004.

[13] D. Bertsimas and A. Thiele, "A robust optimization approach to supply chain management," in International Conference on Integer Programming and Combinatorial Optimization. Springer, 2004, pp. $86-100$.

[14] - "A robust optimization approach to inventory theory," Operations research, vol. 54, no. 1, pp. 150-168, 2006.

[15] P. Brandimarte, "Multi-item capacitated lot-sizing with demand uncertainty," International Journal of Production Research, vol. 44, no. 15, pp. 2997-3022, 2006.

[16] A. Dolgui and J.-M. Proth, Supply chain engineering: useful methods and techniques. Springer Science \& Business Media, 2010.

[17] S. C. Leung and Y. Wu, "A robust optimization model for stochastic aggregate production planning," Production Planning \& Control, vol. 15 , no. 5, pp. 502-514, 2004.

[18] M. Melamed, A. Ben-Tal, and B. Golany, "On the average performance of the adjustable ro and its use as an offline tool for multi-period production planning under uncertainty," Computational Management Science, vol. 13, no. 2, pp. 293-315, 2016.

[19] M. Minoux, "On 2-stage robust lp with rhs uncertainty: complexity results and applications," Journal of Global Optimization, vol. 49, no. 3, pp. 521-537, 2011.

[20] D. Murthy and L. Ma, "Mrp with uncertainty: a review and some extensions," International Journal of Production Economics, vol. 25, no. 1-3, pp. 51-64, 1991.

[21] A. Ouorou, "Affine decision rules for tractable approximations to robust capacity planning in telecommunications," in International Conference on Network Optimization. Springer, 2011, pp. 277-282.

[22] Y. Pochet and L. A. Wolsey, Production planning by mixed integer programming. Springer Science \& Business Media, 2006.

[23] A. L. Soyster, "Convex programming with set-inclusive constraints and applications to inexact linear programming," Operations research, vol. 21, no. 5, pp. 1154-1157, 1973 .

[24] H. M. Wagner and T. M. Whitin, "Dynamic version of the economic lot size model," Management Science, vol. 5, no. 1, pp. 89-96, 1958. 